\title{
Reply: Comment on 'Stage-dependent alterations of the serum cytokine pattern in colorectal carcinoma'
}

\author{
T Kantola ${ }^{1}$, K Klintrup ${ }^{2}$, J P Väyrynen ${ }^{1}$, J Vornanen ${ }^{1}$, R Bloigu ${ }^{3}$, T Karhu ${ }^{4}$, K-H Herzig ${ }^{4,5}$, J Näpänkangas ${ }^{1}$, \\ J Mäkelä ${ }^{2}$, T J Karttunen ${ }^{1}$, A Tuomisto ${ }^{1}$ and M J Mäkinen *,1
}

${ }^{1}$ Department of Pathology, University of Oulu and Oulu University Hospital, Oulu, Finland; ${ }^{2}$ Department of Surgery, Oulu University Hospital, Oulu, Finland; ${ }^{3}$ Medical Informatics Group, University of Oulu, Oulu, Finland; ${ }^{4}$ Institute of Biomedicine and Biocenter of Oulu, University of Oulu, Oulu, Finland and ${ }^{5}$ Department of Psychiatry, Kuopio University Hospital, Kuopio, Finland

Sir,

We thank Dr Guthrie and Professor McMillan (Guthrie and McMillan, 2013) for their interest in our recent publication entitled 'Stage-dependent alterations of the serum cytokine pattern in colorectal carcinoma' (Kantola et al, 2012). They point out additional important aspects relating to the systemic inflammatory response, necrosis and the role of macrophages in serum cytokine response in colorectal cancer, which we did not discuss in detail in our original publication because of the length of the publication. Here, we provide some additional data and analyses on the association of modified Glasgow Prognostic Score (mGPS) to clinical tumour parameters.

Our aim was primarily to detect those cytokines that associate with tumour characteristics. As cytokines act both locally and have distant effects at the systemic level, it is difficult to differentiate the contribution of local, tumour-derived inflammatory and -immune response from systemic reactions and reactions not related to cancer. Therefore, we believed that the utilisation of a panel of cytokines instead of single markers was an advantage to evaluate such alterations in a clinical context. This is supported by our finding that gender, age or location of the cancer did not have any significant effect on the observed cytokine response pattern against cancer that consisted of elevated PDGF, IL6, IL7, IL8 and lower MCP1 levels. At the moment, more studies are needed to find out whether the cytokine alterations in cancer will yield specific advantage for diagnostic and prognostic purposes.

As Guthrie and McMillan point out, systemic inflammatory response - as depicted by the mGPS - was associated with more widespread alterations in serum cytokine levels. It is notable that only $21 \%$ of CRCs (25 out of 116 cases) in our study presented with elevated mGPS (21 cases with mGPS score 1 and 4 cases with score 2), thus the majority of CRCs during their primary operation

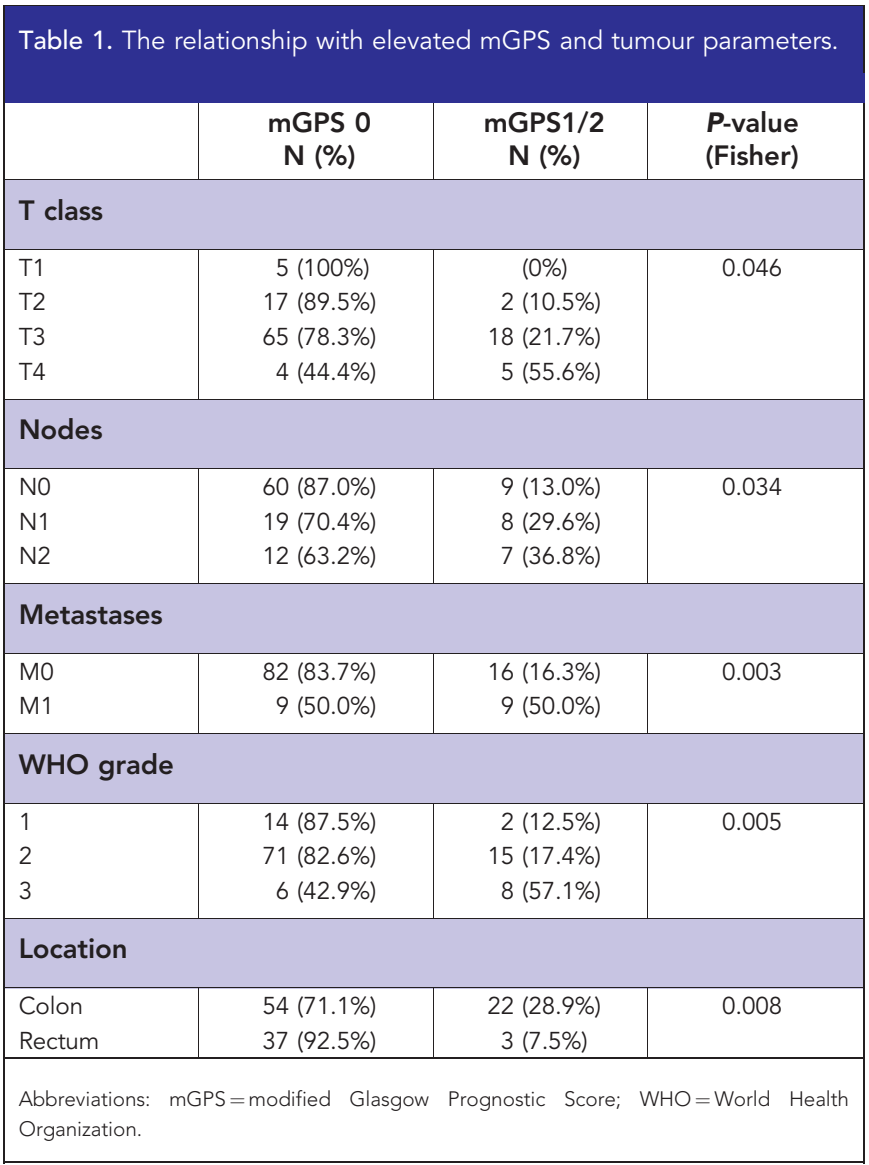


were not associated or did not elicit a significant systemic inflammatory response.

Inspired by the comments of Guthrie and McMillan, we further evaluated the relationship of mGPS to tumour-related and clinical parameters. It is remarkable that elevated mGPS showed significant association with poor differentiation, advancing $\mathrm{T}$ stage, nodal involvement and the presence of metastases as well as tumour location (see Table 1), but not with age, gender or BMI. These findings further strengthen the clinical value of mGPS as a prognostic tool (Roxburgh and McMillan, 2010).

The general observation that curative surgery does not necessarily result in the resolution of systemic inflammatory response suggests that factors other than primary tumour are also involved in eliciting the systemic inflammatory response. There are many possible explanations, but it would be plausible to think that as elevated mGPS shows a significant association with parameters of aggressive behaviour and poor survival, occult dissemination of cancer cells could explain the sustained inflammatory response despite clinically radical surgery.

McMillan and Guthrie suggest that cancer-associated macrophages and necrosis might be important determinants of serum cytokine levels and an important factor linking local and systemic inflammatory responses. Macrophages have a two-sided role in tumour-associated inflammation depending on their location and activation. Tumour-associated macrophages activated towards M2 phenotype are generally considered to promote tumour growth by enhancing angiogenesis, growth, metastasis and immunosuppression (Baay et al, 2011, Hao et al, 2012). We have previously shown that the macrophages surrounding colorectal cancers are independently associated with improved survival (Klintrup et al, 2005). Therefore, we hypothesise that the macrophages associated with tumour necrosis differ from those associating with peritumoural inflammation. It has been recently shown that the amount of M1 macrophages in colorectal cancer is associated with better prognosis irrespective of the amount of M2 macrophages (Edin et al, 2012).

Overall, we agree with Dr Guthrie and Professor McMillan that more detailed studies and follow up is needed to understand more tumour behaviour and to improve our recommendations towards therapy.

\section{REFERENCES}

Baay M, Brouwer A, Pauwels P, Peeters M. Lardon F (2011) tumor cells and tumor-associated macrophages: secreted proteins as potential targets for therapy. Clin Dev Immunol 2011: 565187.

Edin S, Wikberg ML, Dahlin AM, Rutegård J, Öberg Å, Oldenborg PA, Palmqvist R (2012) The distribution of macrophages with a M1 or M2 phenotype in relation to prognosis and the molecular characteristics of colorectal cancer. PLoS One 7: e47045.

Guthrie G, McMillan DC (2013) Comment on 'Stage-dependent alterations of the serum cytokine pattern in colorectal cancer'. Br J Cancer 108: $1915-1916$.

Hao NB, Lü MH, Fan YH, Cao YL, Zhang ZR, Yang SM (2012) Macrophages in tumor microenvironments and the progression of tumors. Clin Dev Immunol 2012: 948098.

Kantola T, Klintrup K, Väyrynen JP, Vornanen J, Bloigu R, Karhu T, Herzig K, Näpänkangas J, Mäkelä J, Karttunen TJ, Tuomisto A, Mäkinen MJ (2012) Stage-dependent alterations of the serum cytokine pattern in colorectal carcinoma. Br J Cancer 107: 1729-1736.

Klintrup K, Mäkinen JM, Kauppila S, Väre PO, Melkko J, Tuominen H, Tuppurainen K, Mäkelä J, Karttunen TJ, Mäkinen MJ (2005) Inflammation and prognosis in colorectal cancer. Eur J Cancer 41: 2645-2654.

Roxburgh CS, McMillan DC (2010) Role of systemic inflammatory response in predicting survival in patients with primary operable cancer. Future Oncol 6: 149-163. 